
\title{
R Reserarch Suare \\ The Quality of Palliative Sedation in End-Stage Disease: Audit From a Department of Oncology and Haematology
}

Saviola Alessia ( $\sim$ saviola.alessia@aou.mo.it)

University Hospital Modena

Schipilliti Francesca Matilde

University Hospital Modena

Isca Chrystel

University Hospital Modena

Massimiliano Salati

University Hospital Modena

Daniele Dini

University Hospital Modena

Claudia Fiorani

University Hospital Modena

Raffaella Postiglione

University Hospital Modena

Flavia Cantile

University Hospital Modena

Leonardo Ferrara

University Hospital Modena

Chiara Carboni

University Hospital Modena

Leonardo Potenza

University of Modena and Reggio Emilia

Massimo Dominici

University of Modena and Reggio Emilia

Mario Luppi

University of Modena and Reggio Emilia

Giuseppe Longo

University Hospital Modena 
Keywords: Palliative sedation, end-stage disease, refractory symptoms, midazolam, Rudkin score, D-PaP score

Posted Date: November 15th, 2021

DOI: https://doi.org/10.21203/rs.3.rs-1047987/v1

License: (1) This work is licensed under a Creative Commons Attribution 4.0 International License. Read Full License

Version of Record: A version of this preprint was published at Supportive Care in Cancer on January 17th, 2022. See the published version at https://doi.org/10.1007/s00520-021-06730-8. 


\section{Abstract}

\section{Purpose}

Palliative sedation (PS) plays a critical role to give suffering relief from refractory symptoms at the end of life. Our audit aimed to assess and improve quality of PS at the Department of Oncology and Hematology of University Hospital of Modena, to verify the adherence to international guidelines, the cooperation among members of care team, focusing with attention on family's perception of this delicate situation.

\section{Methods}

From December 2016 to June 2019, data of patients undergoing PS in the Department were collected by an electronic folder tool, "Sedation Tool" (ST), that recorded clinical and PS informations, D-PaP, Rudkin Score and family's perception.

\section{Results}

245 patients were enrolled. $82 \%$ had a Karnofsky Performance Status $10-20 \%$. The most common cancer types were lung and gastro-intestinal carcinomas ( $27 \%$ and $21 \%$ respectively). Refractory symptoms observed were confusion and agitation (76\%), dyspnea (39\%), pain (15\%), delirium (10\%), psychological distress (5\%). Midazolam was the drug of choice for PS. Most of patients had Rudkin score 5 after 24 hours and $33 \%$ had terminal event within a period of 24 hours from the beginning of PS. During PS most of Patient's relatives reported peacefulness (65\%), agitation/impatience in $6 \%$ of cases, concern for suffering (16\%).

\section{Conclusion}

PS is used in case of worsening general conditions at the end-stage disease to relieve refractory symptoms with dignity. The ST can become a simple instrument to evaluate and improve PS quality, providing more attention on the impact of PS on relatives to then possibly develop new supportive procedures for patients and their families.

\section{Introduction}

Patients with advanced cancer frequently experience different physical and psychological intolerable symptoms in their last weeks of life that are often refractory to standard care treatments. By definition, symptoms which cannot be adequately relieved despite aggressive efforts to identify good therapy are named refractory. Among them, pain, dyspnoea, anxiety, convulsions, delirium are the most common in terminally ill cancer patients [1].

A readiness to address these clinical problems is a medical and moral imperative and palliative care has played a critical role to promote comfort and dignity during the end of life. 
In this clinical context, palliative (or therapeutic) sedation (PS) is a medical intervention advocated by international guidelines as a way to provide suffering relief at the end of life, through the use of medications that induce a state of decreased or absent awareness, without hastening death $[2,6]$, in a manner that is ethically acceptable to the patient, family and health-care providers $[1,3,5]$.

Guidelines are broadly used for regulating and standardising this practice with the aim to improve care, encourage prudence and fill the gap between research and practice. Nonetheless, prevalence and practice pattern of PS vary greatly worldwide and are often tailored to suit localised themes and needs $[3,4]$.

Clinical outcome is certainly considered the most important indicator for PS and to collect information about prognosis, clinical characteristics, patients and families' feeling may be useful for clinicians to better recognize who may eventually require this medical intervention and to improve clinical practice.

Herein we report relevant data about patients undergoing PS, including relatives' perceptions, collected through a PS-specific electronic-based tool filled out by the inpatient staff. The aims of the present study are several-fold. Firstly, to obtain an inner audit about the modality and quality of PS in our Cancer Centre; then, to verify the adherence to the international guidelines on the topic, to ultimately foster the cooperation among members of care team involved, with a particular focus on family's perception of this delicate situation.

\section{Materials And Methods Patients' selection}

We retrospectively reviewed electronic medical records of patients undergoing PS at the Department of Oncology and Haematology of the University Hospital of Modena between December 2016 and June 2019. An electronic folder called "Sedation Tool" with specific items was incorporated into patient records to collect relevant aspects of PS according to the ESMO (European Society for Medical Oncology) and SICP (Società Italiana Cure Palliative)'s recommendations about the management of refractory symptoms at the end of life [Fig. 2].

In particular, the following data were recorded: timing of the PS (first day of treatment and its duration), characteristics of tumours (primary and metastatic sites), chemotherapy (number of previous treatment lines), patient's informed consent, symptoms requiring PS (i.e. delirium, dyspnoea, pain, global suffering, bleeding, vomiting, great epileptic condition), sedative drugs administered (type, initial and final dose), level of sedation of patients (calculated through the Rudkin Score at the beginning, after 6 hours and after 24 hours from PS commencement) and, when used, adjuvant drugs.

Moreover, the electronic tool included variables required to calculate the D-PaP (Delirium Palliative Prognostic) score: dyspnoea, anorexia, KPS (Karnofsky Performance Status), clinical prediction of survival (weeks), total white blood cells (x10^9/L), lymphocyte percentage and delirium. 
Family members' perception regarding the medical intervention underwent by their relatives was finally recorded at the end of the PS path. The inpatient staff, including both physicians and nurses concomitantly, filled out the "Sedation Tool" throughout the entire PS. Then, overall survival (measured in hours) was calculated from the date of PS initiation to the date of death due to any cause.

Audit is a tool for guideline implementation and improvement the quality of care, for these reason there is an acknowledgement of ethical committee and an authorization of Department. Regarding statistical analysis, in descriptive statistics continuous variables were reported as the median and 25-95 percentile, while categorical variables were reported as absolute and percentage frequencies.

\section{Results}

\section{Study population}

A total of 245 inpatients received PS during the study period and were therefore included in the analysis.

The median age was 69 years old (32-96 years) and 154 patients (63\%) were male. The vast majority of them $(n=206,84 \%)$ was affected by solid tumours, with lung cancer as the most common cancer type (27\%), followed by gastrointestinal cancer $(21 \%)$, genitourinary cancer $(10 \%)$, head and neck cancer $(7 \%)$, and breast cancer $(6 \%)$. On the other hand, a total of 39 patients (16\%) were diagnosed with haematological malignancies. Concerning previous treatments for oncological disease 78 patients (32\%) underwent to one line of chemotherapy, $58(24 \%)$ to two lines, $38(15 \%)$ to three lines and $13(5 \%)$ to four or more lines [Table 1].

Most of patients had an end-stage metastatic disease at the time of admission: specifically, 171 (70\%) of them had visceral metastases, $75(31 \%)$ had bone metastasis, and $44(18 \%)$ brain metastases [Table 1].

\section{Characteristics of palliative sedation}

Globally, 18 patients (7\%) gave their informed consensus before the start of PS, whereas $187(76 \%)$ of them were unable to express their wish because of deterioration in their general health conditions.

With regards to clinical reasons for PS, 121 patients (49\%) showed more than one refractory symptom, among which the most common one was confusion/agitation (76\%), followed by dyspnea (39\%), pain $(15 \%)$ and delirium (10\%). In 12 patients (5\%) PS was started for refractory psychological distress and in $3 \%$ of them for an acute intractable episode of hemorrhage or seizure [Table 1].

Overall, general health conditions of patients were compromised: 202 of them (82\%) had in fact a Karnofsky Performance Status (KPS) between $10 \%$ and $20 \%$ showing an overall survival of $1-2$ weeks in $88 \%$ of cases. The analysis of D-PaP score showed a probability of 30 -day survival less than $30 \%$ in 77 patients (31\%), between $30-70 \%$ in 61 patients (25\%) and superior to $70 \%$ in 19 patients ( $8 \%$ ). 
The Rudkin Score analysis, calculated at different consecutive time points, showed $24 \%$ of patients with a score of 1 at the beginning of PS, while most of them had a Rudkin score 5 after 24 hours [Table 2].

Concerning compounds used for PS, benzodiazepines were the drug-class of choice for almost all the treated patients. In particular, midazolam was administered to 231 patients (94\%), and diazepam in 9 patients (4\%). The initial daily dose of midazolam was $15 \mathrm{mg}$ for 142 patients (58\%), between $16-30 \mathrm{mg}$ for 61 patients (25\%) and more than $30 \mathrm{mg}$ for 8 patients (3\%) [Table 3]. Following the initial administration, a dose escalation was undertaken in 98 patients $(40 \%)$.

Morphine was administered as adjuvant drug in 189 patients (77\%) and hydration in 23 patients (9\%).

The analysis of overall survival during sedation (calculated in hours) showed a terminal event within a period of 24 hours from the commencement of PS in 80 patients (33\%), within 24-48 hours in 60 patients $(24 \%)$ and $49-120$ hours in 69 patients (28\%). 31 patients (13\%) survived more than 5 days [Table 4].

\section{Feelings of patients' family members/relatives}

During the course of treatment several interviews were undertaken with patients' relatives, in some cases with the support of a psychologist.

At the end of the PS path, most of patients' relatives reported peacefulness (65\%), followed by concern for suffering $(16 \%)$ and agitation $(6 \%) ; 11 \%$ of them expressed a really satisfaction about the treatment given to their beloved [Fig.1].

\section{Discussion}

Palliative Sedation (PS) has certainly become an important medical intervention for the management of intolerable refractory symptoms in terminally ill cancer patients. In this context, international guidelines used for regulating and standardising this practice are often tailored to suit localised themes and needs of a particular regional/social area $[3,10]$. In this regard, the level of sedation to be reached, drug selection for this practice, the possibility to continue or not life-sustaining therapies, the indication for artificial nutrition and hydration [3, 7], the application of PS in existential distress $[3,4]$ are all example of topics differently covered by guidelines.

Additionally, the exact timing to which PS should be started with respect to patient's life expectancy is another subject of controversy. To this end, the NHPCO (National Hospice and Palliative Care Organization) suggests a prognosis of two weeks or less [10], the EAPC (European Association for Palliative Care) and NCCN (National Comprehensive Cancer Network) refer to "hours or days" [5, 12], while both the AAHPM (American Academy of Hospice and Palliative Medicine) and AMA (American Medical Association) generally refer to the final stages of illness $[13,14]$.

Given the variability in the interventions, in patients' ages, characteristics and needs, more clarity and consistency should be reached in the definition and indications of PS. 
Concerning patients' characteristics, compared to previous experiences that showed a median age of sedated patients from 58 to 67 years with a prevalence of lung and gastro-intestinal cancer $[15,16,17$, $21,22]$, the present study showed a prevalence of elderly patients with $47 \%$ of them aged more than 70 years. Again, roughly half of patients were affected by either lung cancer or gastrointestinal cancer $(27 \%$ and $21 \%$ respectively) at an advanced stage with a high tumour burden and marked deterioration of global health conditions. This largely justifies the low rate of informed consent given by the patients (only $7 \%$ ) and the high rate of consent collected from the family members [Table 1].

In this context, clinical outcome is certainly considered the most important indicator for PS and real-world analysis from palliative care units may be useful for clinicians to improve this delicate practice.

Our retrospective observational study is focused on the evaluation of the pattern and quality of care of PS in our Cancer Centre.

Among the different prognostic scores used in palliative care, we decided to incorporate D-PaP score as prognostic score based on its accuracy in predicting different risk classes for patients survival, as demonstrated by Scarpi and Maltoni $[8,9]$. Then, considering the need to use a simple sedation scale, we employed the Rudkin score to evaluate the efficacy of PS and monitoring the state of consciousness of our patients at different consecutive time points. In accordance with the recommendations coming from international guidelines which underline that the level of sedation should be the lowest necessary to provide adequate relief of suffering, we used midazolam as drug of choice for PS with a starting dose of $15 \mathrm{mg}$ intravenous in 24 hours (58\% of cases). Concurrent administration of parental morphine was common in our population ( $77 \%$ of patients). A dose escalation of midazolam was necessary to maintain the beneficial drug effect in $40 \%$ of cases.

As demonstrated in two multicenter prospective studies $[6,11]$ and in several retrospective analysis [18, 19], deep continuous sedation was not associated with measurable shortening of life, confirming itself as a valid method of managing patients' sufferance in end of life.

In our analysis more than $50 \%$ of patients treated had a survival of longer than 24 hours (13\% more than 5 days). This agrees with median duration of sedation described in other real world analysis: i.e. 48 hours as reported by Schur et al. in their analysis on Austrian patients [15]. Nabal et al. report a mean duration of PS of 1.2 days [17], Hopprich et al. a duration of 27.5 hours before death [23], while Mercadante et al. a median sedation duration of 22 hours [20].

Another matter of debate is certainly the use of PS in event of existential distress because of its strong ethical implication. EAPC framework outlines special considerations for the use of sedation for refractory psychological or existential distress in patients in advanced stages of a terminal illness, including the use of intermittent sedation prior to continuous PS [5]. Other guidelines do not mention this aspect or consider it as not an appropriate response to suffering primarily existential [3, 14]. In our experience, PS should be initiated in cases of severe existential distress only after exclusion of acute psychological deterioration caused by a treatable complication of illness, a reversible metabolic event or medication 
toxicity and after a deep assessment of psychological state of patients, a careful consultation of relatives, psychologist and psychiatrist.

Patient's sufferance and PS are often distressing events for families. Considering the importance to improve the quality of life of patients, but also of families and caregivers, as suggested by IAHPC (International association for Hospice and Palliative Care), an analysis of cultural values, beliefs and relatives' mood should be taken into consideration.

The ST is an easy-to-use electronic device enabling a comprehensive and systematic collection of data pertaining to PS, including families' feeling. Through an user-friendly graphical interface [Fig. 2] it provided us with clear and readily-available information on the topic to be exploited for several purposes. Firstly, it allows a real-time assessment of compliance to PS guidelines by comparing clinical practice measures with scientific society recommendations. Then, the ST aids in standardising medical interventions thanks to a report of data in a constant manner with predefined items, thus potentially reducing the risk for mistakes. Finally, we deemed of particular interest the incorporation of family members feelings within the assessment of our ST, that may help identifying people in need of a tailored psychological support.

Our study has some limitations to be acknowledged, including the retrospective observational study design and the lack in some cases of data derived from an incomplete transcription of them in ST. Despite that, considering the informations collected in ST that include patients clinical characteristics, DPaP score, Rudkin score and relatives' feeling, this can become a simple instrument to evaluate and improve PS quality, personalize treatment of terminal ill patients, providing more attention on the impact of PS on relatives to then possibly develop new supportive procedures for patients and their families.

\section{Conclusion}

PS is a medical intervention used worldwide to give suffering relief from refractory symptoms at the end of life. Guidelines are broadly used for regulating and standardizing this practice, but nowadays there is a lack of a clear algorithm for monitoring patients undergoing to palliative sedation. For this reason analysis from Palliative Care Units may be useful for clinicians to improve this delicate practice, encourage prudence and fill the gap between research and practice. The ST can be a simple instrument to improve PS, personalize treatment according to patient's need and promote new supportive procedures for patients and their families.

\section{Declarations}

Funding: The authors did not receive support from any organization for the submitted work.

Conflicts of interest: The authors have no conflicts of interest to declare that are relevant to the content of this article. 
Availability of data and material: N/A

Code availability: N/A

Authors' contributions: All the authors contributed to the design and implementation of the research, to the analysis of the results and to the writing of the manuscript.

Ethics approval: This is an audit. The University Hospital of Modena Ethics Committee has confirmed that no ethical approval is required.

Consent to participate and Consent to publication: Audit is a tool for guideline implementation and improvement the quality of care, for these reason there is an acknowledgement of ethical committee and an authorization of Department.

\section{References}

1. Cherny NI et al (2014) ESMO Clinical Practice Guidelines for the management of refractory symptoms at the end of life and the use of palliative sedation. Annual of Oncology 25(Supplement 3):iii143-iii152

2. Maltoni $\mathrm{M}$ et al (2012) Palliative sedation in end-of-life care and survival: a systematic review. J Clin Oncol 30(12):1378-1383

3. Gurschick L et at (2015) Palliative Sedation: An analysis of international guidelines and position statements. American Journal of Hospice Palliative Medicine 32(6):660-671

4. Abarshi $E$ et al (2017) International variations in clinical practice guidelines for palliative sedation: a systematic review. BMJ Supportive Palliative Care 7:223-229

5. Cherny $\mathrm{Nl}$ et al (2009) European Association for Palliative Care (EAPC) recommended framework for the use of sedation in palliative care. Palliat Med 23(7):581-593

6. Maeda I et al (2016) Effect of continuous deep sedation on survival in patients with advanced cancer (J-Proval): a propensity score-weighted analysis of a prospective cohort study. Lancet Oncol 17(1):115-122

7. Hui D et al (2015) The Last Days of Life: Symptom Burden and Impact on Nutrition and Hydration in Cancer Patients. Curr Opin Support Palliat Care 9(4):346-354

8. Scarpi E et al (2011) Survival Prediction for Terminally III Cancer Patients. Revision of the Palliative Prognostic Score with Incorporation of Delirium. Oncologist 16(12): 1793-1799

9. Maltoni M et al (2012) Prospective Comparison of Prognostic Scores in Palliative. Care Cancer PopulationsThe Oncologist 17:446-454

10. Kirk TW et al (2010) National Hospice and Palliative Care Organization (NHPCO) position statement and commentary on the use of palliative sedation in imminently dying terminally ill patients. J Pain Symptom Manage 39:914-923 
11. Maltoni $\mathrm{M}$ et al (2009) Palliative Sedation Therapy does not hasten death: results from a prospective multicenter study. Ann Oncol 20:1163-1169

12. National Comprehensive Cancer Network. NCCN clinical practice guidelines in oncology: palliative care (Update 2013) http://www.nccn.org/professionals/physician_gls/pdf/palliative.pdf. Accessed January 2013

13. American Academy of Hospice and Palliative Medicine. Statement on palliative sedation (2013) http://www.aahpm.org/positions/default/sedation.html. Accessed January 2006

14. American Medical Association. Opinion 2.201-sedation to unconsciousness in end-of-life care (2008) ethics/.opinion2201.page. Accessed January 2013

15. Schur S et al (2016) Sedation at the end of life - a nation-wide study in palliative care units in Austria. BMC Palliative Care 1-8

16. Benitez-Rosario MA et al (2012) Quality of Care in Palliative Sedation: Audit and Compliance Monitoring of a Clinical Protocol. J Pain Symptom Manage 44:532e54

17. Nabal M et al (2014) Palliative Sedation: Current Situation and Areas of Improvement. Rev Calid Asist. Mar-Apr 29(2):104-111

18. Rietjens $\mathrm{J}$ et al (2008) Palliative Sedation in a Specialized Unit for Acute Palliative Care in a Cancer Hospital: Comparing Patients Dying With and Without Palliative Sedation. J Pain Symptom Manage 36(3):228-234

19. Maltoni M et al (2012) Palliative Sedation in End-Of-Life Care and Survival: A Systematic Review. J Clin Oncol 30(12):1378-1383

20. Mercadante S et al (2009) Controlled Sedation for Refractory Symptoms in Dying Patients. Controlled Clinical Trial J Pain Symptom Manage 37(5):771-779

21. Turker I et al (2014) Investigational Tests and Treatments Performed in Terminal Stage Cancer Patients in Two Weeks Before Death: Turkish Oncology Group (TOG) Study. Multicenter Study. Med Oncol 31(12):350

22. Boceta $\mathrm{J}$ et al (2013) Palliative Sedation in a University Hospital: Experience After Introducing a Specific Protocol. Rev Calid Asist 28(4):225-233

23. Hopprich A et al (2016) Palliative Sedation at a University Palliative Care Unit-A Descriptive Analysis". Dtsch Med Wochensch 141(8):e60-e66

\section{Tables}

Table 1: Baseline characteristics of study population 


\begin{tabular}{|c|c|}
\hline Clinical characteristics & $\begin{array}{c}\mathrm{N}(\%) \\
245\end{array}$ \\
\hline $\begin{array}{l}\text { Age (years): } \\
\begin{aligned} 32-50 \\
51-70 \\
>70\end{aligned}\end{array}$ & $\begin{array}{l}20(8) \\
110(45) \\
115(47)\end{array}$ \\
\hline $\begin{array}{l}\text { Sex: } \\
\\
\\
\text { Male } \\
\text { Female }\end{array}$ & $\begin{array}{l}154(63) \\
91(37)\end{array}$ \\
\hline $\begin{array}{l}\text { Type of tumor: } \\
\text { Lung } \\
\text { Gastrointestinal } \\
\text { Head and Neck } \\
\text { Genito-urological } \\
\text { Breast } \\
\text { Cerebral } \\
\text { Hematological } \\
\text { Other }\end{array}$ & $\begin{array}{l}66(27) \\
52(21) \\
17(7) \\
25(10) \\
14(6) \\
13(5) \\
39(16) \\
19(8)\end{array}$ \\
\hline $\begin{array}{c}\text { Site of metastasis: } \\
\text { Visceral } \\
\text { Bone } \\
\text { Brain } \\
\text { Not reported }\end{array}$ & $\begin{array}{l}171(70) \\
75(31) \\
44(18) \\
53(22)\end{array}$ \\
\hline $\begin{array}{c}\mathrm{N}^{\circ} \text { of metastatic sites: } \\
\text { No metastases } \\
1 \text { site } \\
\geq 2 \text { sites } \\
\quad \text { Not reported }\end{array}$ & $\begin{array}{l}2(1) \\
108(44) \\
82(33) \\
53(22)\end{array}$ \\
\hline $\begin{array}{c}\mathrm{N}^{\circ} \text { chemotherapy lines: } \\
\text { No chemotherapy } \\
1^{\text {st }} \text { line } \\
2^{\text {nd }} \text { line } \\
3^{\text {rd }} \text { line } \\
4^{\text {th }} \text { or more lines }\end{array}$ & $\begin{array}{l}58(24) \\
78(32) \\
58(24) \\
38(15) \\
13(5)\end{array}$ \\
\hline $\begin{array}{c}\text { Patient informed consent: } \\
\text { Yes } \\
\text { Unworkable } \\
\text { Not reported }\end{array}$ & $\begin{array}{l}18(7) \\
187(76) \\
40(16)\end{array}$ \\
\hline $\begin{array}{l}\text { Symptoms: } \\
\text { Confusion and agitation } \\
\text { Dyspnea } \\
\text { Pain } \\
\text { Delirium } \\
\text { Psychological distress } \\
\text { Hemorrhage }\end{array}$ & $\begin{array}{l}186(76) \\
95(39) \\
36(15) \\
25(10) \\
12(5) \\
4(2)\end{array}$ \\
\hline
\end{tabular}




\begin{tabular}{|c|l|}
\hline Epileptic attack & $2(1)$ \\
\hline KPS (\%): & $202(82)$ \\
$10-20$ & $30(12)$ \\
$30-40$ & $1(1)$ \\
$>50$ & $12(5)$ \\
Not reported & \\
\hline Expected survival (weeks): & $216(88)$ \\
$1-2$ & $12(5)$ \\
$3-4$ & $4(1)$ \\
$5-6$ & $1(1)$ \\
$>6$ & $12(5)$ \\
Not reported & \\
\hline probability of 30 days survival (\%) & \\
30-70\% (B) & \\
> $70 \%$ (A) & $77(31)$ \\
Not reported & $61(25)$ \\
& $19(8)$ \\
& $88(36)$ \\
\hline D-PaP - A,B,C): & \\
\hline
\end{tabular}

Abbreviations: KPS (Karnofsky performance status); PaP (Palliative Prognostic Score) Some patients showed more than one symptom requiring Palliative sedation.

Table 2: Rudkin score at the beginning of PS and after 6 and 24 hours from its commencement

\begin{tabular}{l|l|l|l|}
\hline \multicolumn{4}{l|}{ Rudkin Score (Noof patients, \%) } \\
\hline & T0 & $6 \mathrm{~h}$ & $24 \mathrm{~h}$ \\
\hline 1 & $59(24)$ & $4(2)$ & $3(1)$ \\
\hline 2 & $23(9)$ & $14(6)$ & $5(2)$ \\
\hline 3 & $36(15)$ & $29(12)$ & $7(3)$ \\
\hline 4 & $17(7)$ & $28(11)$ & $17(7)$ \\
\hline 5 & $10(4)$ & $32(13)$ & $55(22)$ \\
\hline Not reported & $100(41)$ & $138(56)$ & $158(65)$ \\
\hline & & & \\
\hline
\end{tabular}

Abbreviation: PS (Palliative sedation)

Table 3: Type of drugs and doses used for sedation 


\begin{tabular}{|l|l|}
\hline Variable & $\mathbf{N}(\%)$ \\
\hline Drug: & $231(94)$ \\
Midazolam & $9(4)$ \\
Diazepam & $1(<1)$ \\
Delorazepam & $2(1)$ \\
Morphine & $2(1)$ \\
Not reported & \\
\hline Initial dose of Midazolam (mg): & $23(9)$ \\
< 15 & $142(58)$ \\
15-30 & $61(25)$ \\
31-45 & $6(2)$ \\
>45 & $2(1)$ \\
Not reported & $10(4)$ \\
10 & $1(1)$ \\
Initial dose Diazepam (mg): & \\
\hline Dose Variation: & $110(45)$ \\
None & $98(40)$ \\
Increase & $37(15)$ \\
Not reported & \\
\hline
\end{tabular}

Table 4: Survival during palliative sedation (Hours)

\begin{tabular}{|l|l|}
\hline Survival (hours) & $\mathbf{N}^{\circ}(\%)$ \\
\hline$<24$ & $80(33)$ \\
\hline $24-48$ & $60(24)$ \\
\hline $48-120$ & $69(28)$ \\
\hline$>120$ & $31(13)$ \\
\hline Not reported & $5(2)$ \\
\hline
\end{tabular}

Figures 


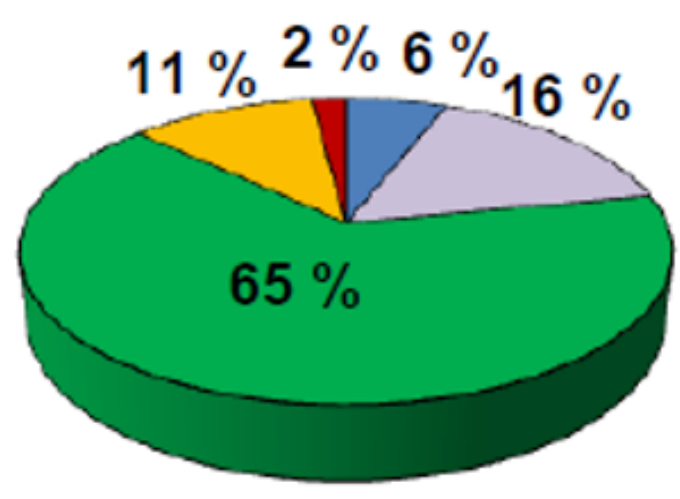

$\square$ Agitation-Impatience

口Concern for suffering

口Peacefulness

$\square$ Satisfaction about treatment

口Other

\section{Figure 1}

Patients' relatives feeling during Palliative sedation 


\section{SEDATION}

Start 03/04/2019

Cancer type: Bladder cancer

Metastatic gites: bone, lung, prostate

Line of chemotherapy: I

Consenting patient: yes

Symptoms: pain

D-PaP Score: less than $30 \%$

Rudkin Score: T0 1, 6h 3, $24 \mathrm{~h} 5$.

Drug: midazolam; Dosage: $15 \mathrm{mg} / 24 \mathrm{~h}$

Adjuvant drug: morfine

Hours from initiation sedation to end: 50

Patient relatives' feeling: concern for suffering

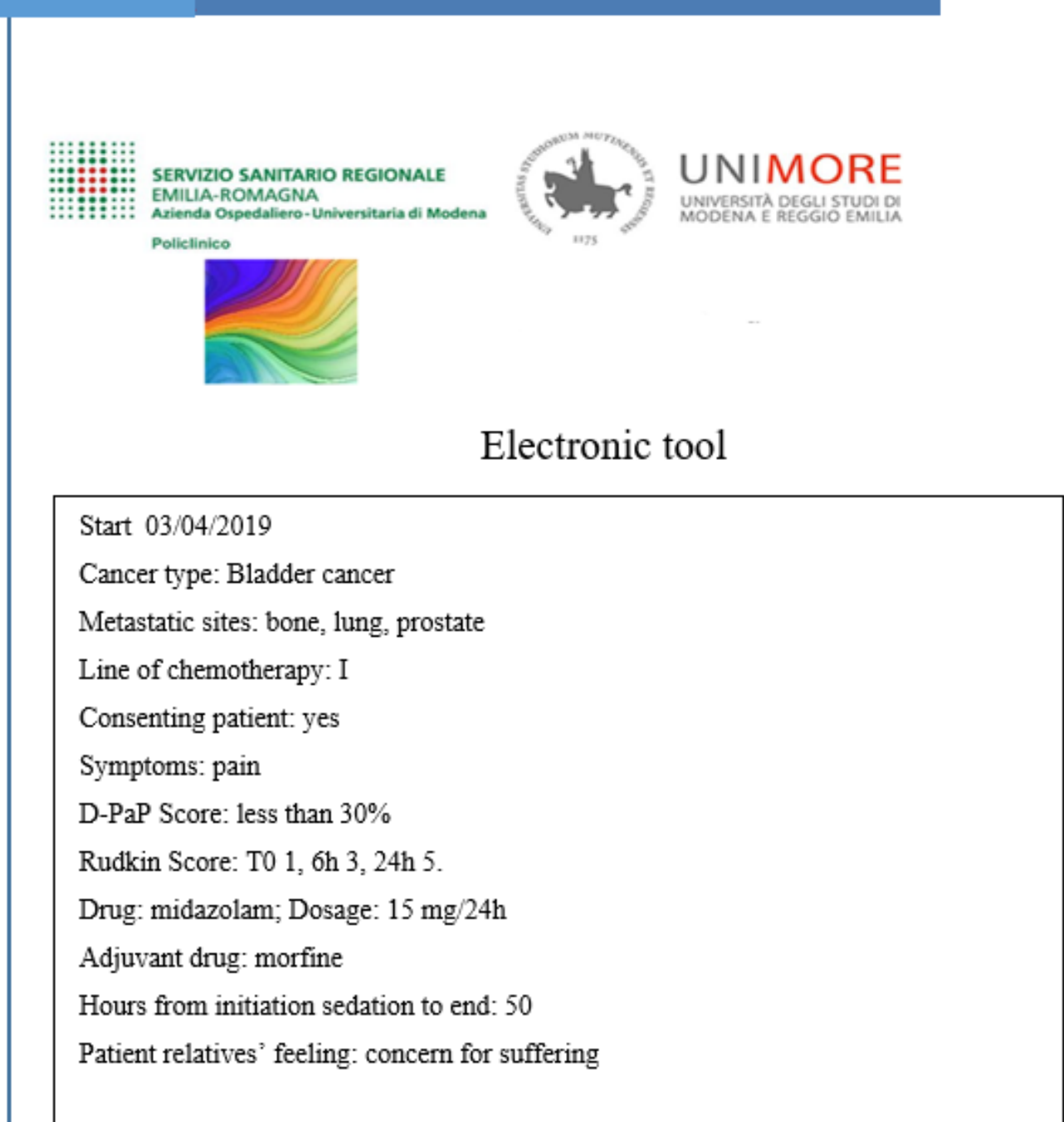

\section{Figure 2}

\section{Sedation tool}

